\title{
Multi-wavelength Continuum
}

\section{Emission of AGN}




\title{
THE ORIGIN OF CONTINUUM EMISSION IN ACTIVE
}

\author{
GALACTIC NUCLEI
}

\author{
JOEL N. BREGMAN \\ Department of Astronomy, University of Michigan
}

\begin{abstract}
The general understanding of the continuum emission from AGN has changed from the picture where nonthermal processes were responsible for all of the emission. The current body of observation indicates that there are two types of objects, one being the blazar class (or blazar component), where nearly all of the emission is nonthermal, due primarily to synchrotron and inverse Compton emission. Variability studies indicate that the emitting region decreases with size from the radio through the $\mathrm{X}$-ray region, where the size of the $\mathrm{X}$-ray region is of order a light hour. More than two dozen of these radio-loud AGNs have been detected at GeV energies (one source at $\mathrm{TeV}$ energies), for which the radiation mechanism may be inverse Compton mechanism.

In the other class, the radio-quiet AGN (component), the emission is almost entirely thermal, with radiation from dust dominating the near infrared to submillimeter region. The optical to soft X-ray emission is often ascribed to black body emission from an opaque accretion disk, but variability studies may not be consistent with expectations. Another attractive model has freefree emission being responsible for the optical to soft X-ray emission. The highest frequencies at which these AGN are detected is the $\mathrm{MeV}$ range, and these data should help to determine if this emission is produced in a scattering atmosphere, such as that around an accretion disk, or by another model involving an opaque pair plasma.
\end{abstract}

\section{Introduction}

There has been steady progress in understanding the continuum emission from active galactic nuclei (AGNs), although the present picture is, in some respects, considerably different than it was a decade ago. This review examines how the models have changed, presents my view of the current picture, and outlines a few of the remaining issues facing the field.

Prior to about 1980, it was widely believed that, aside from a variety of emission lines and some line recombination radiation, all of the emission from AGN was nonthermal in origin. This point of view developed from the strong evidence that there was abundant nonthermal emission from radio-loud objects, combined with the philosophical belief that all AGNs were fundamentally similar. This philosophical belief of a single type of object, which might be attributed to Occam's Razor, was shown to be too simple by a wealth of data that accumulated in the 1980's.

\section{The Radio Quiet AGNs}

\subsection{Emission From Dust}

The two most important changes that occurred in the 1980's were the detailed measurements of the submillimeter through infrared region, and the study of the continuum emission over a very large wavelength region. In the application of these techniques, perhaps the greatest surprise occurred in the study of the radio-quiet 
AGNs, such as the Seyfert galaxies and the radio-quiet quasars. These objects are not entirely radio-silent, as they are detected by the sensitive radio telescopes of today. However, the ratio of the radio to optical fluxes is orders of magnitude less than for the radio-loud AGNs, such as the BL Lac objects and the radioloud quasars. Despite the relative weakness of their radio emission, the radioquiet AGNs were often powerful sources of infrared emission, even at the longest wavelengths accessible to the Infrared Astronomical Satellite (IRAS), $100 \mu \mathrm{m}$. The transition from a far infrared flux density $\left(3 \times 10^{12} \mathrm{~Hz}\right)$, measured in $\mathrm{Jy}$, to the radio emission $\left(10^{10} \mathrm{~Hz}\right)$, measured in $\mathrm{mJy}$, necessitates a rapid decrease in the flux density with decreasing wavelength.

A similar sharp drop from powerful far infrared emission to weak radio emission was seen in an entirely different class of extragalactic object, starburst galaxies (e.g., Soifer, Houck, and Neugebauer 1987; Telesco 1988). However, the emission from these galaxies is successfully modeled by thermal dust emission of absorbed optical and ultraviolet light rather than by nonthermal mechanisms. In the thermal dust model, the sharp decrease in the flux density occurs because dust becomes transparent at wavelengths longward of 50-100 $\mu \mathrm{m}$ and no longer emits as a blackbody (Draine 1990). The falloff can be extremely rapid, although the precise shape depends on grain composition, size, and temperature.

The synchrotron process can also produce a sharp falloff in the continuum emission, so it is necessary to try to distinguish between the dust and synchrotron models. For synchrotron emission, the low frequency falloff occurs as the plasma becomes opaque to its own radiation. In a homogeneous plasma, the opaque region has a slope of $F_{\nu} \propto \nu^{5 / 2}$ below some critical frequency that depends upon magnetic field, density and size of the region. Since these properties might be expected to vary amongst individual objects, the critical frequency should be seen by observers over a range of frequencies in an ensemble of sources.

These predictions were tested by observations that spanned the radio, millimeter, submillimeter, and far infrared regions (e.g., Chini et al. 1988, 1989; Barvainis and Antonucci 1989; Hughes et al. 1993; also, these proceedings). These researchers found that the spectral slope in the falloff region was often steeper than $5 / 2$ (average slope of 3.75; Hughes et al. 1993), which violates the most extreme model with synchrotron emission (inhomogeneous synchrotron emitting regions without a sharp outer boundary always would produce a slope shallower than 5/2). Furthermore, the falloff occurred at approximately the same rest wavelength, for sources with a wide range of luminosities, and this falloff region was at approximately the same wavelength as in starburst galaxies. A characteristic turnover frequency is predicted from the dust model (near $100 \mu \mathrm{m}$ ), where dust becomes transparent. Such studies firmly established that thermal dust emission was the primary continuum mechanism in the submillimeter through infrared range for the radio-quiet AGNs.

This understanding led to renewed interest in developing models for dust emission. A general property of these models is that there must be a range of tempera- 
tures in the emitting dust region in order to fit the observed continuum. The dust temperature is determined by the balance between the absorbed optical-UV light from the AGN nucleus and the radiative rate of the dust grains (provided that the grains are not very small; Rees et al. 1969; Penston et al. 1974). Consequently, the warmest dust grains are closest to the central source (typically 0.1-1 pc distance), while the coolest dust grains, those responsible for the 60-100 $\mu \mathrm{m}$ emission, must lie 1-10 kpc from the nucleus (Barvainis 1987). This far infrared emitting region is so large, that it should have appeared to be non-variable during the year when the IRAS satellite was making measurements. An analysis of the 60-100 $\mu \mathrm{m}$ IRAS fluxes from radio-quiet AGNs confirmed this prediction of no variability (Clement et al. 1987; Edelson and Malkan 1987). At the shorter wavelengths, variability would be detectable, but the amplitude would be expected to decrease with increasing wavelength, which was seen by Cutri et al. (1985). Also, there would be a time delay between an outburst in the core region (the optical-UV region) and the reradiated emission (the near IR region). This time delay is of particular value because it provides valuable information on the size of the emitting region, for which there is a prediction. Such time delays are observed: in Fairall 9 the dust is thought to lie in the 0.3-1.3 light year region (Clavel, Wamsteker, and Glass 1989; Barvainis 1992); in NGC 3783, and 80-90 day lag is seen between the near-IR and UV regions (Glass 1992); in GQ Comae, a 250 day time delay is seen between the optical and near infrared regions (Sitko et al. 1993). These time delays are close to expectations, further supporting the dust model and the predicted spatial distribution.

Multifrequency observations of radio-quiet AGNs revealed a second important feature, a relative minimum in the power per logarithmic bandpass occurring at a rest frame wavelength of about $1 \mu m$ (e.g., Sanders et al. 1989; Hughes et al. 1993). This feature fit into the dust picture naturally, as it is linked to the sublimation temperature for dust. At temperatures exceeding $1000 \mathrm{~K}$, dust begins to sublimate, a process that proceeds rapidly at temperatures closer to $2000 \mathrm{~K}$. Dust close enough to the central source is heated to these temperature and quickly destroyed, so the emission from them is expected to be minimal. At these temperatures, dust behaves like a blackbody, so $2000 \mathrm{~K}$ corresponds to $1.5 \mu \mathrm{m}$, the approximate wavelength at which the minimum in the power occurs.

There is an alternative view of the origin of the near infrared emission in radioquiet AGNs, a nonthermal power-law origin, although any support for this view is shrinking. Such a power-law is desirable to those fitting accretion disk models to the optical-UV emission (e.g., Sun and Malkan 1989), and it gathers its only real support by the near infrared to X-ray correlation that is seen (Edelson and Malkan 1986; Carleton et al. 1987). However, the near infrared emission is contaminated by galaxy light, so the correlation should improve if the galaxian contribution were removed. Such a test was performed by Kotilainen et al. (1992), who found that, in Seyfert 1 galaxies, the correlation did not improve, in conflict with expectation for the power-law picture. 


\subsection{The Big Blue Bump}

The emission from the optical to the beginning of the soft X-ray emitting region is often referred to as the Big Blue Bump because, when plotting power per logarithmic bandwidth, there is a prominent local maximum in the ultraviolet region, with a noticeable falloff at shorter and longer wavelengths (e.g., Kolman et al. 1993). Usually, this is not just a local maximum, but an absolute maximum in the power output of these systems. If one defines this Big Blue Bump as extending from 1 $\mu m$ to $0.5 \mathrm{keV}$, it contains typically one-third or one-half of the entire observed power from the object; the second most important energetic region is usually the infrared to submillimeter region. Some of the emission in the Big Blue Bump region is due to broad and narrow emission lines, a forest of Fe II emission lines, plus hydrogen recombination radiation. However, these contribute only modestly to the total power, most of which is attributed to some other feature or structure, such as an accretion disk.

Thermal emission from an accretion disk seemed to be the natural explanation for the Big Blue Bump, although recent observations call into question this explanation. One of the most powerful arguments for an accretion disk is theoretical in that it is should occur naturally and is an efficient mechanism through which energy can be extracted as material falls into the gravitational potential of a putative black hole (Rees 1984). Efforts to test this model observationally are concerned with comparing data to predictions of emission from the opaque surface, or to a possible atmosphere above the surface of the accretion disk. The observed Big Blue Bumps can be fit with thermal emission from an accretion disk, provided that one assumes an underlying nonthermal power-law for the near infrared emission (Sun and Malkan 1989; Malkan 1991). Since the near infrared emission is due to dust, the assumed nonthermal power-law continuum should be discarded. It may be possible to fits accretion disk models along with dust emission models, but current efforts seem to require a dust temperature that may be in excess of the maximum expected value (Malkan 1989).

Other tests of emission from accretion disks fail to provide confirmation of the model, but they do not rule out the model either. Accretion disks are not solid bodies, but have a vertical extent with a decreasing gas density distribution, creating a type of photosphere for which the actual structure is highly modeldependent. Hydrogen continuum absorption shortward of 912 A would be expected for a wide range of models, but this phenomenon is not detected (Antonucci, Kinney, and Ford 1989). If this disk and atmosphere are observed in any orientation other than face-on, the continuum emission should be polarized. Polarization at the $1-2 \%$ range is observed, and while that was originally believed to be below the expected values, more accurate predictions, including the effects of general relativity, indicate that it is consistent with the data (Laor, Netzer, and Piran 1990; Netzer 1991).

An important inconsistency between models of accretion disks and the data 
deals with the time delay between variations seen in various wavelength bands. In all opaque accretion disk models, the emitting region at a particular wavelength is associated with a particular radius, with the hottest regions (shortest wavelength) closest to the center. Then, a disturbance near the center of the accretion disk would cause an outburst at short wavelengths, and this disturbance might propagate radially outward at the local sound speed, causing an outburst at longer wavelengths at some later time. For the typical black hole masses and accretion disks discussed for AGNs, the time delay between UV and optical variations should be weeks. However, in the few systems for which measurements of the delays have been attempted, such as NGC 5548, no time delay has been measured, with upper limits of a few days (Couroisier and Clavel 1991; Molendi, Maraschi, and Stella 1992).

Efforts to find periodic behavior in the light curve, associated with rotation of or through the accretion disk, has been dealt a severe blow with recent ROSAT observations of NGC 6814 (see Madjeski, these proceedings). They show that the 12,000 second periodic variability is not intrinsic to the central engine of NGC 6814 but is due to an X-ray binary star system in the Galaxy that was close on the sky to NGC 6814 so that the two systems were confused when observed with previous X-ray instruments.

The only other evidence in support of orbital motion in the cores of AGNS is the double-peaked line profiles, a signature of rotating material, seen in generally radio-loud objects, such as 3C390.3 or 3C 332 (Chen, Halpern, and Fillipenko 1989; Halpern 1990). The double- peaked lines are seen to vary in 3 C390.3, and continued observations are warranted to determine if there is any validity to the 10.4 year "sinusoidal" variations reported by Veilleux and Zheng (1991).

Given the difficulties with sustaining the standard accretion disk model, perhaps the model should be strongly modified or entirely discarded (e.g., Celotti, Fabian, and Rees 1992). One of the most attractive alternative suggestions is that optically thin free-free radiation dominates the observed optical-ultraviolet continuum (detailed discussion in Barvainis 1993; also Ferland, Korista, and Peterson 1990; Malkan and Sargent 1982). In this model, the shape of the $1 \mu \mathrm{m}$ to $0.1 \mu \mathrm{m}$ continuum is adequately fit without requiring an underlying power-law or very hot dust. Also, this model solves the time-delay problem, discussed above. There would be no time delay between an ultraviolet and optical outburst, because freefree emission occurs over a broad wavelength band, unlike blackbody radiation.

For this model to be successful, the continum emitting region must be small enough to be consistent with the observed variations. However, it appears possible to make the emitting region small enough and still retain the transparent nature of the emission, with an optical depth that may be as great as 0.1 .

A complete "cartoon" of the emitting region must be able to relate the behavior at various wavebands, and critical to this picture are the $\mathrm{X}$ - rays. As discussed by Clavel (these proceedings) for the case of NGC 5548, the optical-UV region does not show strong variability on timescales of less than 1 day, while the X-ray emission 
can vary strongly to a timescale of 0.1 days. This suggests that the $\mathrm{X}$-rays come from a region smaller (interior to) the optical-UV region. Furthermore, the two regions are closely related as seen in the correlated variability between the X-ray and optical-UV bands.

Then, in our "cartoon", the X-ray emission lies closest to the black hole and may be largely opaque. Exterior to this emitting region is the free-free emitting region, which may be a dense extended atmosphere around an accretion disk. The freefree emitting region is heated by the photons and possibly fast particles from the $\mathrm{X}$-ray region. Depending upon the geometry, the free-free region (as an accretion disk atmosphere) may be transparent when seen face-on, but nearly opaque as seen edge-on, which would be its orientation to the $\mathrm{X}$-ray emission region, making absorption of the X-rays an efficient process.

\subsection{The X-RAY Emission}

The X-ray emission, which spans three orders of magnitude in energy from 0.1-100 $\mathrm{keV}$ is a complex region with several phenomena present (see review by Mushotzky, Done, and Pounds 1993). At the low energy end of this band, the 0.1-1 keV region, the spectrum is falling rapidly and is probably the high frequency extension of the Big Blue Bump (e.g., Masnou et al. 1992; Turner et al. 1993). This feature, referred to as the "soft excess", appears to be present in most, if not all radio-quiet AGNs (Walter and Fink 1993; Shastri et al. 1993).

The emission above $1 \mathrm{keV}$ is adequately fit by a power-law component, Fe $\mathrm{K}$ emission, plus Fe reflected continuum emission (Pounds, Nandra, and Stewart 1992). Upon removing the contribution from $\mathrm{Fe}$, the underlying power-law has a slope (energy index) of 0.9-1.0. This power-law continuum extends to about 100 $\mathrm{keV}$, at which point, a spectral break is seen (see summary of OSSE observations by Kurfess, these proceedings).

Two distinct models for this power-law continuum have become popular, a pair cascade model and a Compton scattering model (reviewed by Zdziarski, these proceedings). In the first model, the emission emerges from a region that is at least partly opaque to photon-photon collisions, which mediate electron-positron pair production (e.g., Svensson, R. 1987; Done and Fabian 1989; Zdziarski et al. 1990). In the second model, the $\mathrm{X}$-ray emission is produced by Compton scattering of soft photons by a thermal population of hot electrons, such as might occur in an accretion disk corona (Maraschi and Molendi 1991; Walter and Courvoisier 1992; Haardt and Maraschi 1993). The latter model was first developed to explain certain $\mathrm{X}$-ray properties of Galactic black hole binaries, for which there are some spectral similarities to AGNs (Tanaka 1992). The critical test of the model will come from studies at energies near the electron-positron annihilation line $(0.511 \mathrm{MeV})$, where the two models make significantly different predictions for continuum shape and annihilation line strength. 


\section{Blazars}

\subsection{Is It All Nonthermal Emission?}

In contrast to the radio-quiet AGNs, where our understanding changed radically during the past decade, recent observations of radio-loud objects have reaffirmed the picture that developed in the mid-1970s (Jones, O'Dell, and Stein 1974). That is, nonthermal synchrotron emission dominates the radio through ultraviolet region, and inverse Compton scattering probably dominates the hard X-ray and gamma-ray regions.

To assure ourselves that nonthermal processes are required by the observations, we examine the "purest" of the radio-loud AGNs, the BL Lac objects. The reason that the radio data are believed to be nonthermal synchrotron emission is that the brightness temperature is commonly $10^{11}-10^{12} \mathrm{~K}$ in the core, requiring relativistic electron (e.g., Gabudza, Wardle, and Roberts 1989). Furthermore, the emission is highly polarized, power-law in shape, and there is evidence for ordered magnetic fields, all consistent with synchrotron theory; finally, relativistic bulk motion is required. The radio spectrum extends smoothly into the submillimeter, infrared, and optical region, suggesting that they have an origin in synchrotron emission (e.g., Browne et al. 1989a,b; Bregman et al. 1990). In the far infrared, an estimate of the brightness temperature can be derived from the limited variability observations made with IRAS, which has a characteristic timescale shorter than typical radio variations but longer than optical variations (Edelson and Malkan 1987). These observations suggest a brightness temperature greater than $10^{6} \mathrm{~K}$, well above the temperature that dust can radiate at (recall that dust is the source of the far infrared emission in the radio-quiet AGNs), but consistent with a synchrotron origin. At higher frequencies, optical data exhibit rapid variability and high polarization (Carini and Miller 1992; Carini et al. 1992; Moore and Stockman $1981,1984)$, and the soft X-ray data has shown variability so rapid that it occurs on a time scale comparable to or shorter than the light crossing time of a massive black hole, suggesting the presence of relativistic motion (Doxsey et al. 1983; Feigelson et al. 1986; Giommi et al. 1986). These observations of BL Lac objects (and Blazars in general) provide either direct evidence for, or consistency with the synchrotron process over approximately ten orders of magnitude in frequency space (more detailed review in Bregman 1990).

The identification of continuum features was a key to understanding radio-quiet AGNs, so it is worth looking for such features in the blazar class of objects. The general nature of the continuum is that the power per logarithmic bandwidth rises from the radio, peaking somewhere in the millimeter to ultraviolet band. The peak is generally quite broad and its location peak has an enormous range in the rest wavelength (Landau et al. 1986; Browne et al. 1989a,b; Bregman 1990). This range in continuum characteristics suggest a sizable range in the underlying properties of the emitting relativistic plasma.

A distinctive feature has been seen in the infrared-UV region of some BL Lacs, 
a sharp high frequency turnover in the continuum that has been attributed to an upper energy cutoff to the electron distribution (Rieke et al. 1979, 1982; Beichman et al. 1981a,b; Bregman et al. 1981). The identification of this feature is best achieved in the infrared-UV range, where instrumental sensitivity is good, but for some objects, this feature may occur in the extreme UV or X-ray region as well. Giommi (these proceedings) suggests that the difference between radio and $\mathrm{X}$-ray selected BL Lacs is that for the X-ray selected BL Lacs, the cutoff does not occur until the $\mathrm{X}$-ray or higher frequency regions; the other, and possibly more common view, is that the two groups of objects possess different orientations of their relativistically boosted beams with respect to our line of sight (Maraschi et al. 1986; Padovani and Urry 1992; Padovani 1992).

The BL Lac objects show virtually no evidence for thermal emission from gas (except a few exceedingly weak emission lines), but some of the objects in the blazar class possess the same spectral features as were seen in the radio-quiet AGNs (e.g., Sanders et al. 1989). These objects seem to be a combination of the pure nonthermal objects (BL Lacs) and the radio-quiet thermally emitting sources. The relationship between these two type of sources is one of the major problems that remains to be clarified. Such crossover objects are the most complex to study, and it is somewhat unfortunate that the brightest quasar in the sky, 3C 273, is this type of object. The study of AGN continuum emission might have progressed in a more direct manner if the brightest and first objects studied had been a classical BL Lac object (i.e., OJ 287, BL Lac) and a typical radio-quiet AGN (i.e., the Seyfert 1 galaxy NGC 5548).

\subsection{ReLATIVISTIC Jets}

Early VLBI observations of the central core of the radio emission established the asymmetric nature of the central emitting region, and subsequent observations have shown that the emission is confined to a narrow channel (jet-like) on lengthscales spanning orders of magnitude $\left(1-10^{5} \mathrm{pc}\right.$; Bridle and Perley 1984). These observations led to the development of models for the radio region, which also have been applied to the higher frequency emission, where a relativistic shock propagates along an inhomogeneous plasma jet. One of the most impressive successes of this model has been the ability to reproduce the radio outbursts, seen in several frequencies, in objects such as BL Lac (Hughes, Aller, and Aller 1989a,b; Hughes, these proceedings).

The optical and X-ray emitting regions are believed to originate in a jet, but on a size scale smaller than the radio emitting region (Ghisellini et al. 1986, 1993; Marscher, Gear, and Travis 1992). The relative sizes are suggested by the shape of the continuum, but more convincingly by the variability studies. The typical timescale of substantial variability is months-years in the radio region, days in the optical region, and hours in the X-ray region (review in Bregman 1990).

The relative sizes of one emitting region with respect to another can be investigated by examining correlated variability and time delays. In the transparent 
region, such as the infrared through X-ray wavebands, the synchrotron emission from an ensemble of electrons is broad-band, which may make it difficult to determine time delays. In a variability study of PKS 2155-304 (Edelson et al. 1994; these proceedings), the X-ray variations are correlated with and leads those in the ultraviolet by 2 hours, clearly establishing the connection between the two regions and the estimate for the size. Formally, no lag is seen between the UV, optical, and infrared regions, with an upper limit to the lag of 1 day (a similar limit is found for BL Lac; Bregman et al. 1990), although there is evidence that the ultraviolet variations precede those in the optical region (Urry et al. 1993). There is a modest correlation between optical and radio variations, with a time delay that is typically a year, but determining such correlations is hampered because of the different statistical nature of the variability in the two bands (Hufnagel and Bregman 1992).

\subsection{High ENERgy EmisSion}

When the X-ray emission appears to be an extension of the infrared- opticalultraviolet continuum, it is likely to be synchrotron emission from the inner part of the jet (i.e., PKS 2155-304; Edelson et al. 1994). However, for those objects where the $\mathrm{X}$-ray emission is not an extrapolation of the lower frequency synchrotron continuum, another emission mechanism must be active, such as the inverse Compton process.

There is, as yet, no definitive proof that the inverse Compton process produces such high frequency emission, but there are some observations that offer support. A good correlation might be expected between the scattered (X-ray) and the seed photons (infrared-millimeter), and a good correlation is seen (Owen, Helfand, and Spangler 1981). Additionally, the fluxes of the seed and scattered photons would be expected to vary together, and such correlated variations are seen, in a limited data set, for BL Lac (Bregman et al. 1990; Kawaii et al. 1991).

Perhaps the most exciting new development at high energies comes from the EGRET instrument on the Compton Gamma-Ray Observatory, which can measure fluxes in the $\mathrm{GeV}$ photon range. As of the time of this meeting, EGRET has detected 26 AGNs, and every one is a radio-loud object (one of these sources, Mrk 421 is detected in the $\mathrm{TeV}$ range by the Whipple telescope; Punch et al. 1992); not a single radio- quiet object is detected in the $\mathrm{GeV}$ range (see the contributions by Kurfess and Thompson, these proceedings). The best correlation between the $\mathrm{GeV}$ emission and the continuum at lower frequencies is with the radio emission: all detected sources are at least $0.5 \mathrm{Jy}$ at $5 \mathrm{GHz}$. This astonishing correlation is suggestive of an upscattering process, such as the inverse Compton mechanism, which is a promising explanation (Marscher and Bloom 1992; Bloom and Marscher 1993).

Variability has been seen at these gamma-ray energies in 3C 279 (Kniffen et al. 1993), and the timescale of variation (a few days) is characteristic of the opticalultraviolet region, although it would be impossible to detect very rapid variations 
at gamma-ray energies because of the extremely low count rates.

In the distribution of the emitted power, it is difficult to determine whether the gamma-rays may is the dominant power source or only a modest contributor. If the Doppler boosting factor is the same at all frequencies so that the observed flux density distribution is a true representation of the power distribution, then some of the sources detected by EGRET are dominated by gamma-ray emission (e.g., 3C454.3; Hartman et al. 1993). However, the sources detected in gamma- rays may represent only transient outburst events. It is equally important to examine which of the optically and radio bright sources were not detected and the implications for the power distribution. For example, the bright source BL Lacertae has not been detected by EGRET, which implies that the gamma-ray contribution to the total power is less than that from the submillimeter through optical region. Finally, a definitive discussion of the power distribution is hampered by the uncertainty of the magnitude of the Doppler boosting parameter in the gamma-ray region, relative to the lower frequency emitting regions. With the continued operation of GRO, we look forward to what should be rapid progress in this field and an elucidations of many of these important issues.

JNB wishes to acknowlege support from NASA under grant NAGW-2135.

\section{References}

Antonucci, R.R.J., Kinney, A.L., and Ford, H.C. 1989, Ap. J., 342, 64.

Barvainis, R. 1987, Ap. J., 320, 537.

Barvainis, R. 1992, Ap. J., 400, 502.

Barvainis, R., and Antonucci, R. 1989, Ap. J. Sup. Ser., 70, 173.

Barvainis, R. 1993, Ap. J., 412, 513.

Beichman, C.A., Neugebauer, G., Soifer, B.T., Wootten, H.A., Roellig, T., and Harvey, P.M. 1981a, Nature, 293, 711.

Beichman, C.A., Provdo, S.H., Neugebauer, G., Soifer, B.T., Matthews, K., and Wootten, H.A. 1981b, Ap. J., 247, 780.

Bloom, S.D., and Marscher, A.P. 1993, in Proceedings of the Compton Observatory Symposium, ed. N. Gehrels (New York: AIP), in press.

Bregman, J.N. 1990, Astr. Ap. Rev., $2,125$.

Bregman, J.N., et al. 1981, Nature, 293, 714.

Bregman, J.N., et al. 1990, Ap. J., 352, 574.

Bridle, A.H., and Perley, R.A. 1984, Ann. Rev. Astr. Ap., 22, 319.

Browne, L.M.J., et al. 1989a, Ap. J., 340, 129.

Browne, L.M.J., et al. 1989b, Ap. J., 340, 162.

Carini, M.T., and Miller, H.R. 1992, Ap. J., 385, 146.

Carini, M.T., and Miller, H.R., Noble, J.C., and Goodrich, B.D. 1992, Astr. J., $104,15$.

Carleton, N.P., et al. 1987, Ap. J., 318, 595.

Celotti, A., Fabian, A.C., and Rees, M.J. 1992, M.N.R.A.S., 255, 419.

Celotti, A., Maraschi, L, and Treves, A. 1991, Ap. J., 377, 403.

Chen, K., Halpern, J.P., and Filippenko, A.V. 1989, Ap. J., 339, 742.

Chini, R., Steppe, H., Kreysa, E., Krichbaum, T., Quirrenbach, A., Schalinski, C., and Witzel, A. 1988, Astr. Ap., $192, \mathrm{~L} 1$.

Chini, R., Kreysa, E., and Biermann, P.L. 1989, Astr. Ap., $219,87$.

Clavel, J., Wamsteker, W., and Glass, I.S. 1989, Ap. J., 337, 236.

Clement, R., Sembay, S., Hanson, C.G., and Coe, M.J. 1988, M.N.R.A.S., $230,117$. 
Couroisier, T.J.-L, and Clavel, J. 1991, Astr. Ap., 248, 389.

Cutri, R.M., Wisniewski, W.Z., Rieke, G.H., and Lebofsky, M.J. 1985, Ap. J., 296, 423.

Done, C., and Fabian, A.C. 1989, M.N.R.A.S., 240, 81.

Doxsey, R. et al. 1983, Ap. J., 264, L43.

Draine, B.T. 1990, in The Interstellar Medium in Galaxies, ed. H.A. Thronson, and J.M. Shull (Kluwer: Dordrecht),p. 483.

Edelson, R.A., and Malkan, M.A. 1986, Ap. J., 308, 59.

Edelson, R.A., and Malkan, M.A. 1987, Ap. J., 323, 516.

Edelson, R.A., et al. 1994, Ap. J., in press.

Feigelson, E.D., et al. 1986, Ap. J., 302, 337.

Ferland, G.J., Korista, K.T., and Peterson, B.M. 1990, Ap. J., 363, L21.

Gabuzda, D.C., Wardle, J.F.C., and Roberts, D.H. 1989, Ap. J., 336, L59.

Ghisellini, G., Maraschi, L., Tanzi, E., Treves, A. 1986, Ap. J., 310, 317.

Ghisellini, G., Padovani, P., Celotti, A., and Maraschi, L. 1993, Ap. J., 407, 65.

Giommi, P., et al. 1986, Ap. J., 303, 596.

Glass, I.S., 1992, M.N.R.A.S., 256, 23P.

Haardt, F., and Maraschi, L. 1991, Ap. J., 380, L51.

Halpern, J.P. 1990, $A p$. J., 365, L51.

Hartman, R.C. et al. 1993, Ap. J., 407, L41.

Hufnagel, B.R., and Bregman, J.N. 1992, Ap. J., 386, 473.

Hughes, D.H., Robson, E.I., Dunlop, J.S., and Gear, W.K. 1993, M.N.R.A.S., 263, 607.

Hughes, P.A., Aller, H.D., Aller, M.F. 1989a, Ap. J., 341, 54.

Hughes, P.A., Aller, H.D., Aller, M.F. 1989b, Ap. J., 341, 68.

Jones, T.W., O’Dell, S.L., and Stein, W.A. 1974, Ap. J., 188, 353.

Kawai, N. et al. 1991, Ap. J., 382, 508.

Kniffen et al. 1993, Ap. J., 411, 133.

Kolman et al. 1993, Ap. J., 402, 514.

Kotilainen, J.K., Ward, M.J., Boisson, C., DePoy, D.L., and Smith, M.G. 1992, M.N.R.A.S., 256, 149.

Landau, R., et al. 1986, Ap. J., 308, 78.

Laor, A., Netzer, H., and Piran, T. 1990, M.N.R.A.S., 242, 560.

Malkan, M. 1989, in Theory of Accretion Disks, ed. F. Meyer, W. Duschl, J. Frank, and E. MeyerHofmeister (Kluwer: Dordrecht), p. 19.

Malkan, M. 1991, in Structure and Emission Peoperties of Accretion Disks, ed. C. Bertout, S. Collin, J.-P. Lasota, J. Tran Thanh Van, (Gif sur Yvette: Ed. Frontiers), p. 165.

Malkan, M.A., and Sargent, W.L.W. 1982, Ap. J., 254, 22.

Maraschi, L., Ghisellini, G., Tanzi, E., Treves, A. 1986, Ap. J., 310, 325.

Maraschi, L., and Molendi, S. 1991, Ap. J., 368, 138.

Marscher, A.P., and Bloom, S.D. 1992, in Compton Observatory Science Workshop, p. 346.

Marscher, A.P., Gear, W.K., and Travis, J.P. 1992, in Variability of Blazars, ed. E. Valtaoja and M.J. Valtonen (Cambridge: CUP), p. 85.

Masnou, J.L., Wilkes, B.J., Elvis, M., McDowell, J.C., and Arnaud, K.A. 1992, Astr. Ap., 253, 35.

Molendi, S., Maraschi, L., and Stella, L. 1992, M.N.R.A.S., 255, 27.

Moore, R.L., and Stockman, H.S. 1981, Ap. J., 243, 60.

Moore, R.L., and Stockman, H.S. 1984, Ap. J., 279, 465.

Mushotzky, R.F., Done, C., and Pounds, K.A. 1993, Ann. Rev. Astr. Ap., 31, 717.

Netzer, H. 1991, in Structure and Emission Properties of Accretion Disks, IAU Colloquium No. 129 , p. 177.

Owen, F.N., Helfand, D.J., and Spangler, S.R. 1981, Ap. J., 250, L55.

Padovani, P. 1992, Astr. Ap., 256, 399.

Padovani, P., and Urry, C.M. 1992, Ap. J., 387, 449.

Penston, M.V., Penston, M.J., Selmes, R.A., Becklin, E.E., and Neugebauer, G. 1974, M.N.R.A.S., $169,357$.

Pounds, K.A., Nandra, K., and Stewart, G.C. 1992 in Ginga Memorial Symposium, ed. F. Makino 
and F. Nagase (ISAS: Tokyo), p. 45.

Punch, M. et al. 1992, Nature, 358, 477.

Rees, M.J., Silk, J.I., Werner, M.W., and Wickramsinghe, M.C. 1969, Nature, 223, 788.

Rees, M.J. 1984, Ann. Rev. Astr. Ap., 22, 471.

Rieke, G.H., Lebofsky, M.J., and Kinman, T.D. 1979, Ap. J., 232, L151.

Rieke, G.H., Lebofsky, M.J., Wisniewski, W.Z. 1982, Ap. J., $263,73$.

Sanders, D.B., Phinney, E.S., Neugebauer, G., Soifer, B.T., and Matthews, K. 1989, Ap. J., 347, 29.

Shastri, et al. 1993, Ap. J., 410, 29.

Sitko, M., Sitko, A.K., Siemiginowska, A., and Szczerba, R. 1993, Ap. J., 409, 139.

Soifer, B.T., Houck, J.R., and Neugebauer, G. 1987, Ann. Rev. Astr. Ap., 25, 187.

Sun, W.-H., and Malkan, M.A. 1989, Ap. J., 346, 68.

Svensson, R. 1987, M.N.R.A.S., 227, 403.

Tanaka, Y. 1992 in Ginga Memorial Symposium, ed. F. Makino and F. Nagase (ISAS: Tokyo), p. 19.

Telesco, C.M. 1988, Ann. Rev. Astr. Ap., 26, 26.

Turner, J. et al. 1993, Ap. J., 407, 556.

Urry, C.M., et al. 1993, Ap. J., 411, 614.

Veilleux, S., and Zheng, W. 1991, Ap. J., 377, 89.

Walter, R., and Courvoisier, T.J.-L. 1992, Astr. Ap., 266, 65.

Walter, R., and Fink, H.H. 1993, Astr. Ap., 274, 105.

Zdziarski, A.A., Ghisellini, G., George, I.M., Svensson, R., Fabian, A.C., Done, C. 1990, Ap. J., 363, L1.

Zdziarski, A.A., and Krolik, J.H. 1993, Ap. J., 409, L33. 